\title{
Das Transparenzgebot als Kontrollmaßstab Allgemeiner Geschäftsbedingungen
}

\author{
INGO KOLLER
}

\section{Problem}

1. Die neueste Rechtsprechung zum Transparenzgebot

Im sog. Annuitätendarlehens-Urteil' hat der BGH die Auffassung vertreten, daß eine Klausel, die an sich inhaltlich angemessen ist, dann gegen $\$ 9$ AGBG verstößt, wenn den Kunden des AGB-Verwenders die preiserhöhende oder sonst benachteiligende Wirkung der Klausel erst nach intensiver Beschäftigung mit ihr oder aufgrund ergänzender Auskünfte deutlich wird. Treu und Glauben verpflichten nach Ansicht des BGH den AGB-Verwender, die Rechte und Pflichten seiner Vertragspartner möglichst klar und durchschaubar darzustellen. Dieser Standpunkt wurde im Wertstellungsurteil ${ }^{2}$ bestätigt. Der BGH bejahte in diesem Urteil eine inhaltliche Unangemessenheit der Wertstellungsbestimmungen. Er ließ es offen, ob anders zu entscheiden wäre, wenn intransparente Klauseln üblich wären und zur Verkehrssitte geworden wären. Voraussetzung für eine Verkehrssitte sei jedenfalls, daß sich die Vertragspartner der in den intransparenten Klauseln enthaltenen Regelungen bewußt seien, im Ergebnis also auch faktische Transparenz. In die Richtung auf das formale Transparenzgebot geht auch die Erwägung, daß „fingierte Zinsrechnungsfaktoren ... nur hingenommen werden (können), wenn dabei die Belange des Kunden in angemessener Weise - zum Beispiel durch Angabe des Effektivzinses (...)- berücksichtigt werden“ ${ }^{\text {“ }}$.

\section{Die Kritik dieser Rechtsprechung}

Bruchner ${ }^{4}$ und Wagner-Wieduwilt ${ }^{5}$ haben gegen die Art und Weise, in der in diesen beiden Entscheidungen das Transparenzgebot eingesetzt

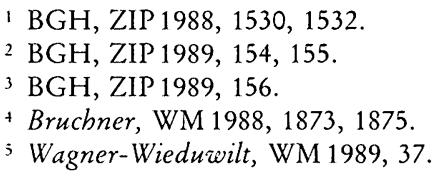


worden ist, eindringlich Kritik angemeldet. Ihrer Meinung nach läßt sich weder aus den Gesetzesmaterialien noch aus der bisherigen Rechtsprechung und Literatur ein Transparenzgebot des Inhalts herleiten, daß eine Klausel im Sinn des $\$ 9$ AGBG unangemessen ist, wenn ein aus der Klauselgestaltung resultierender Nachteil, der für sich gesehen materiellrechtlich durchaus angemessen ist, für den Kunden nicht ohne weiteres erkennbar ist.

In der Tat mutet es seltsam an, daß sämtliche AGB so ausgestaltet sein sollen, daß der Kunde vor Vertragsschluß die auf ihn zukommenden Nachteile ohne weiteres erkennen kann, obwohl sich Kunden typischerweise und sinnvollerweise nicht die Mühe machen, auch nur einen Blick auf die AGB zu werfen. Daraus resultiert ja unter anderem ${ }^{6}$, wie in der juristischen Diskussion allgemein vertreten wird ${ }^{7}$, die besondere Gefährdung der Kunden durch AGB. Dies wurde im Rahmen der ökonomischen Analyse des AGBG genauer herausgearbeitet.

\section{Das Transparenzgebot im Licht der ökonomischen Analyse}

a) Posner 8 meint, die Tatsache, daß Unternehmen sich nahezu immer weigern, in Verhandlungen über einzelne Klauseln von Standardverträgen einzutreten, sei nicht Ausdruck der Ohnmacht der Vertragsgegenseite. Wenn der AGB-Verwender nicht den Markt beherrsche, so werde der Kunde zu einem Konkurrenten gehen, der dem Kunden bessere Konditionen anbieten werde. Der Wettbewerbsdruck sorge dafür, daß sich die Verbesserung der Konditionen bis zu einem optimalen Marktgleichgewicht fortsetze. Adams $s^{9}$ hat dem in einer eingehenden ökonomischen Analyse des AGBG entgegengehalten, daß Posner zwar zutreffend die Angemessenheit von Vertragskonditionen nicht von der Möglichkeit des Aushandelns abhängig mache. Ausreichender Wettbewerbsdruck sorge zwar schon dann für ein optimales Marktgleichgewicht, wenn Kunden zu besseren Angeboten abwandern können und der Anbieter

' Hönn, Kompensation gestörter Vertragsparität (1982), S. $147 \mathrm{ff}$; zur Restriktion des $\$ 9$ AGBG, wenn der Kunde die Bedeutung und Tragweite einer Klausel voll erfaßt hat Roussos, JZ 1988, 997, 1002; dagegen Niedenführ, Informationsgebote des AGB-Gesetzes (1986), S. $15 \mathrm{ff}$ m. Nachw.

7 G. Roth, ÖZW 1977, 36; Wolf in Wolf/Horn/Lindacher, AGBG (1. Aufl.) $\$ 3$ AGBG Rdn. 1; Dauner-Lieb, Verbraucherschutz durch Ausbildung eines Sonderprivatrechts für Verbraucher (1983), S.72; Hönn, Kompensation gestörter Vertragsparität (1982), S. 150; G. Stein, Die Inhaltskontrolle vorformulierter Verträge des allgemeinen Privatrechts (1982), S.41; Niedenführ, Informationsgebote des AGB-Gesetzes (1986), S. $11 \mathrm{ff}$ mit weiteren Nachweisen.

\& Posner, Economic Analysis of Law (2. Aufl.), $\$ 4.8$.

"Adams in: Ansprüche, Eigentums- und Verfügungsrechte, Hrsg. v. Manfred Neumann, Schriften des Vereins für Sozialpolitik 1983, neue Folge Bd. 104. 
dies weiß. In der Tat ist es wenig vernünftig, aus dem Fehlen von Verhandlungen beim Kauf ohne weiteres einen Schluß auf eine Marktstörung zu ziehen. Man denke nur an den Kauf von Lebensmitteln im Supermarkt. Posner hat jedoch, wie Adams herausarbeitet, bei der Beurteilung der AGB-Problematik die Kosten der Informationsgewinnung und Informationsverarbeitung zu wenig berücksichtigt.

b) Ein richtiges Verständnis von $A G B$ ist typischerweise mit einem außerordentlichen Aufwand verbunden. Nicht nur der Leseaufwand ist häufig beträchtlich. Hinzu kommt, daß sich die volle inhaltliche Bedeutung der einzelnen Klauseln meist nur bei mehrmaligem Lesen erschließt, bei dem jedes Wort gewendet und genau auf die grammatikalischen Bezüge geachtet werden muß. Der durchschnittliche Kunde soll der Rechtsprechung zufolge auch Sinn und Zweck der auszulegenden Klausel berücksichtigen ${ }^{10}$. Er soll die Klausel darüber hinaus im Licht ihrer systematischen Stellung deuten ${ }^{11}$, ja wie verschiedentlich angenommen wurde, sogar die Entstehungsgeschichte einer Klausel kennen und beachten. Von manchen wird auch angenommen, daß der Kunde juristische Begriffe in ihrem Inhalt richtig erkennen können solle ${ }^{12}$. Es kommt daher nicht von ungefähr, daß zu weit verbreiteten $A G B-W e r k e n$, wie den ADSp, den Bankbedingungen oder der VOB, umfangreiche Kommentierungen existieren ${ }^{13}$. Aber selbst wenn man nicht auf derartige umfangreiche AGB abhebt und vom Kunden nicht erwartet, daß er Sinn und Zweck einer Klausel durchdenkt und der systematischen Stellung sowie Entstehungsgeschichte der Klausel Rechnung trägt, so bleiben doch die Informationskosten und das Informationsrisiko des Kunden beträchtlich; denn der Kunde muß sich nicht nur die Bedeutung jeder Klausel genau überlegen. Er läuft auch das Risiko, daß ihm später entgegengehalten wird, seine Auslegung habe nicht dem Verständnis redlicher Durchschnittsvertragspartner unter Abwägung der Interessen der an Geschäften dieser Art beteiligten Kreise entsprochen. Im Wissen um den hohen Prüfungsaufwand und die Unsicherheit der Prüfungsergebnisse werden Kunden daher typischerweise bei Vertragsschluß auf

10 BGHZ22, 109, 113; BGH, NJW 1968, 885; BB 1981, 452; kritisch Ulmer in Ulmer/Brandner/Hensen, AGBG (5. Aufl.) $\$ 5$ Rdn. 21.

11 BGH, NJW 1977, 379, 381; 1979, 2148, 2149; 1985, 320, 324; kritisch Ulmer (Fn. 10) $\$ 5$ Rdn. 21.

12 Palandt/Heinrichs, BGB (48. Aufl.), $\$ 5$ AGBG Rdn. 3; Erman/Hefermehl, BGB (7. Aufl.), $₫ 5$ AGBG Rdn. 4; a. A. Ulmer (Fn. 10) $₫ 5$ Rdn. 19; Lindacher in Wolf/Horn/ Lindacher, AGBG (1. Aufl.), $₫ 5$ Rdn. 10; Münchener Kommentar-Kötz, BGB (2. Aufl.), $\$ 5$ AGBG Rdn. 3.

$13 \mathrm{Vgl}$. auch BGH, NJW 1985, 623, 625 zu einem 170 Seiten starken Formularvertrag. 
die nähere Prüfung selbst relativ kurzer vorformulierter Vertragsklauseln verzichten ${ }^{14}$. In großem Umfang enthalten ja AGB erfahrungsgemäß Regelungen für Ereignisse wie Leistungsstörungen, deren Eintrittswahrscheinlichkeit als gering veranschlagt werden muß. Da einer Erhöhung des Prüfungsaufwands nicht im gleichen Umfang ein präziseres Wissen um den Vertragsinhalt gegenübersteht, minimieren Kunden normalerweise ihre Kosten wirtschaftlich effizient dadurch, daß sie auf eine Überprüfung der AGB bei Vertragsschluß gänzlich verzichten oder sie nur ganz oberflächlich überfliegen. Dieses Verhalten ist nach Inkrafttreten des AGBG sogar einzig wirtschaftlich und vernünftig. Der Kunde kann nämlich im Grundsatz davon ausgehen, daß die AGB keine materiell unangemessenen Klauseln enthalten, und wenn sie derartige Klauseln enthalten sollten, kann er darauf vertrauen, daß sie nicht zum Tragen kommen. Wie Rabe $e^{15}$ kürzlich festgestellt hat, wäre es oft sogar taktisch falsch, im Weg von Vertragsverhandlungen auf die Eliminierung unzulässiger AGB zu dringen.

Insgesamt gesehen ist es also wirtschaftlich wenig sinnvoll, zu fordern, daß die Kunden immer schon bei Vertragsschluß in der Lage sein müssen, die ihnen im Rahmen der AGB auferlegten Nachteile ohne weiteres, ja bei oberflächlichem Lesen auf den ersten Blick hin in ihrer Tragweite zu durchschauen.

c) Das soll nicht bedeuten, daß die Forderung nach transparenten AGB wirtschaftlich gänzlich unsinnig wäre. Sie hat im Gegenteil durchaus ihre Berechtigung. Nur besteht der umfassende Transparenzbedarf eben nicht so sehr im Zeitpunkt des Vertragsschlusses, sondern vielmehr in dem Moment, in dem es zu Streitigkeiten gekommen ist oder Streitigkeiten bevorstehen. In dieser Phase des Vertrages ist es von großer Bedeutung, daß die AGB übersichtlich aufgebaut, lesbar und verständlich sind. Der Kunde soll dann, wenn er sich aus Anlaß einer konkreten Auseinandersetzung die Mühe macht, die AGB genau zu überprüfen, einigermaßen schnell die maßgebliche Regelung ausfindig machen und die Rechtslage eigenständig zuverlässig ermitteln können. Ziel der Transparenz auf dieser Vertragsabwicklungsstufe ist daher die Vermeidung von Prozessen. Der Kunde soll außerdem davor geschützt werden, daß er seine Rechte nicht wahrnimmt, weil er sie nicht oder nicht richtig erkennen kann und ohne die Beiziehung eines Rechtsanwalts resigniert.

it Dauner-Lieb (Fn.7), S.72f, die mit großer Plausibilität ausführt, daß Aufklärungspflichten die Informationsdefizite nicht beseitigen könnten.

is D. Rabe, NJW 1987, $1978 \mathrm{ff}$. 


\section{Analyse der Rechtsprechung zum Transparenzgebot}

a) Soweit die Rechtsprechung mit dem Topos der Transparenz bzw. der Durchschaubarkeit operiert, ist vielfach nicht klar erkennbar, ob es ihr um die Klarstellung der Rechtslage nach Vertragsschluß oder um die Verhandlungs- bzw. Markttransparenz vor Vertragsschluß oder um beides geht. Eingehender wird zu diesen Fragen, soweit ersichtlich, nirgends Stellung genommen.

b) Die überwiegende Zahl der Urteile sucht wohl mit Hilfe des Transparenzgebotes den Entscheidungsspielraum des AGB-Verwenders einzuschränken und dem Kunden ein schlagkräftiges Instrument für die ex post-Kontrolle von Ermessensentscheidungen des AGB-Verwenders an die Hand zu geben. In diese Kategorie fallen die Urteile zur Angemessenheit von Änderungsvorbehalten ${ }^{16}$ sowie zur Berechnung der Ausgleichszahlungen beim Leasing ${ }^{17}$. So führt der BGH in seiner Entscheidung vom 11.6.1980'8 aus, es gelte zu verhindern, daß es im Einzelfall zu gerichtlichen Auseinandersetzungen komme oder daß der Betroffene eine Preiserhöhung hinnehme, weil sich das zulässige Ausmaß der Preiserhöhung nicht anhand der akzeptierten AGB beurteilen lasse ${ }^{19}$. Ähnlich wird auch in dem Urteil vom 12.6.198520 darauf hingewiesen, daß der Leasingnehmer für den Fall der Kündigung erkennen können muß, welche Ausfälle und Nachteile der Leasinggeber in seine Berechnung einbeziehen dürfe und ob die Vorteile des Leasinggebers infolge der Kündigung berücksichtigt worden sind. Außerdem müsse die Abzinsungsmethode offenbart werden. Da kein Leasingnehmer sich für den hypothetischen Fall der Kündigung eingehend mit deren Folgen befassen wird, kann dies nur bedeuten, daß dem Leasingnehmer die ex post-Kontrolle über die Sachgerechtigkeit der Abrechnung eröffnet sein soll, wenn er tatsächlich gekündigt hat und eine Abrechnung erhält. In der Entscheidung vom 24.11.198421 scheint der Gedanke der Begrenzung des Ermessensspielraums im Vordergrund zu stehen, wenn die Angemessenheit eines Änderungsvorbehalts von dessen Konkretisierung nach Voraussetzungen und Umfang abhängig gemacht wird ${ }^{22}$. Der BGH hält nämlich ein Bestimmungsrecht im Rahmen des billigen Ermessens für unangemessen, obwohl eine derartige Klausel bei Vertragsschluß

16 BGH, NJW 1985, 623, 627; NJW 1980, 2518.

17 BGH, NJW 1985, 2253, 2255; 1982, 870.

18 NJW 1980, 2518.

19 Ebenso BGH, NJW 1985, 320, 321; OLG Koblenz, WM 1984, 1259.

20 BGH, NJW 1985, 2252, 2253; ebenso NJW 1982, 870; 1987, 379.

21 BGH, NJW 1985, 623, 627.

22 BGH, NJW 1984, 1182, 1183. 
sicherlich äußerst transparent ist; denn der Kunde, der die AGB studiert hat, weiß, daß dem AGB-Verwender ein äußerst weiter Ermessensspielraum eröffnet wird. Daß der Spielraum nicht fest umrissen ist, macht die Klausel selbst nicht intransparent. Gefährlich für den Kunden ist allein der ex post schwer kontrollierbare Ermessensspielraum des AGB-Verwenders.

c) In der jüngsten Zeit finden sich vermehrt Urteile, in denen die Gerichte eine Information der Kunden durch AGB ex ante, d.h. bei Vertragsschluß, sicherstellen wollen. So heißt es im Urteil des OLG Karlsruhe vom 23.4.1986 ${ }^{23}$, dem Leasingnehmer müsse klar und eindeutig bewußt werden, daß seine Entgeltverpflichtung nicht auf die Zahlung der Leasingraten während der Mietzeit beschränkt sei, sondern daß er im Fall eines Mindererlöses Zusatzzahlungen zu leisten habe. Der Leasinggeber habe daher auf der Vorderseite des Vertrages eine transparente Darstellung dieser Pflicht zu geben. Im Annuitätendarlehens-Urteil ${ }^{2+}$ ergibt sich der ex ante Standpunkt daraus, daß nach Ansicht des BGH die Intransparenz der Regelung weggefallen wäre, wenn die Bank den Effektivzins angegeben hätte. Da der Effektivzins nur beim Preisvergleich vor dem Vertragsschluß eine wesentliche Rolle spielt, ist es klar, daß es dem BGH mit dem Transparenzgebot um die Angebotstransparenz bei Vertragsschluß ging. Noch deutlicher tritt diese neue Position im Wertstellungs-Urteil25 zutage, wo ausdrücklich formuliert wird: "Treu und Glauben verpflichten die Verwender allgemeiner Geschäftsbedingungen, die Rechte und Pflichten ihrer Vertragspartner eindeutig und verständlich darzustellen, damit diese sich bei Vertragsschluß hinreichend über die rechtliche Tragweite der Vertragsbedingungen klar werden können." Auch hier wird betont, daß Transparenz durch Angabe des Effektivzinses oder durch offen ausgewiesene Gebühren geschaffen werden könne.

d) Diese beiden Urteile fallen, wie Wagner-Wieduwilt ${ }^{26}$ zu Recht betont, aus der Reihe der übrigen BGH-Urteile zum Transparenzgebot heraus; denn hier wird erstmals eindeutig dem Transparenzgebot eine Funktion bei Vertragsschluß zugewiesen ${ }^{27}$. Dieser These stehen auch nicht die Reihe von Urteilen zur Transparenz entgegen, in denen es nicht so deutlich wird, daß für Transparenz erst nach Vertragsschluß im Vorfeld von Rechtsstreitigkeiten gesorgt werden soll. Wenn etwa der

23 NJW-RR 1986, 1112.

24 Siehe Fn. 1.

25 Siehe Fin. 2.

26 Siehe Fn. 5.

27 Anders in der Tendenz Köndgen, NJW 1989, 943, $944 \mathrm{f}$. 
$\mathrm{BGH}$ einen Vertrag für sittenwidrig hält ${ }^{28}$, weil die $\mathrm{AGB}$ nicht klar formuliert oder unübersichtlich geordnet sind, oder wenn er eine Klausel, die "handelsübliche" Abweichungen vom Muster für zulässig erklärt, für ausreichend transparent hält ${ }^{29}$, so kann dies dem Schutz des Kunden sowohl vor als auch nach Vertragsschluß dienen ${ }^{30}$. Wie unklar die Tragweite des Transparenzgebotes in der BGH-Rechtsprechung ist, zeigen auch die Urteile vom 11.11.1968 und vom 29.9.19832. Im erstgenannten Urteil postuliert der $\mathrm{BGH}$ einerseits, daß Klauseln unwirksam seien, falls sich der Kunde über die uneingeschränkte Annahme entscheiden müsse, ohne sich wegen des Umfangs oder des schwer zu verstehenden Inhalts über die Tragweite der Klauseln klar werden zu können. Andererseits betont der BGH in diesem Urteil, daß der Kunde überfordert wäre, wenn von ihm verlangt werden würde, daß er vor Unterzeichnung des Vertrages den gesamten Inhalt der Urkunde überprüft. Also nimmt der BGH an, daß der Kunde bei Vertragsschluß allenfalls einen Teil der Klauseln überprüft. In Hinblick auf den Rest bedarf es dann aber keiner Transparenz bei Vertragsschluß. Im Urteil vom 29.9.1983 33 ging es um einen Vertrag, der zu einem Pauschalpreis abgeschlossen worden war. In dem Formularvertrag wurde ein Recht statuiert, zusätzliche Aufschließungskosten, die bereits bekannt waren, zu berechnen. Der BGH meinte, daß diese Klausel auch dann unangemessen sei, wenn der Notar den Kunden über diese Klausel belehrt habe, weil die Klausel unredlich versteckt worden sei. Das Motiv, die Preiserhöhung zu verstecken, nicht aber die Intransparenz, die ja durch die Notarbelehrung wegfallen sollte, läßt die Klausel als unangemessen erscheinen.

e) Insgesamt läßt sich festhalten, daß der Einsatz des Transparenzgebotes im Moment des Vertragsschlusses nicht eindeutig auf eine ständige Rechtsprechung gestützt werden kann. Vielmehr spricht vieles dafür, daß der $\mathrm{BGH}$ mit dem Annuitätendarlehen $\mathrm{s}^{-34}$ und Wertstellungsurtei ${ }^{35}$ dem Transparenzgebot eine neue Dimension eröffnet hat. Ob dies im Einklang mit dem AGBG stand und auch rechtlich sinnvoll war, ist im Folgenden zu prüfen.

28 BGH, WM 1982, 1354, 1359.

29 BGH, NJW 1987, 1886, 1887.

30 Ebenso BGH, NJW 1985, 53, 56; NJW 1983, 159, 162; 1983, 1322, 1325.

31 NJW 1969, 230, $232 \mathrm{f}$.

32 NJW 1984, 171.

33 Siehe Fn. 32.

${ }^{3+}$ Siehe Fn. 1.

35 Siehe Fn. 2. 


\section{Das Informationsmodell im Rahmen des AGBG}

Das AGBG geht davon aus, daß der Wettbewerb nicht in der Lage ist, für angemessene $A G B$ und so für Vertragsgerechtigkeit zu sorgen. In einer Situation, in der die Marktkräfte nicht mehr in der Lage sind, einen angemessenen Interessenausgleich herbeizuführen, stehen grundsätzlich zwei Modelle zur Verfügung, um die unangemessene Verfolgung einseitiger Interessen zu erschweren, wenn nicht zu verhindern. Das eine Modell kann als Informationsmodell bezeichnet werden. Ihm liegt die Annahme zugrunde, daß die möglicherweise durch Vertragskonditionen benachteiligten Vertragspartner nur ausreichend über den Inhalt der Vertragsbedingungen aufgeklärt werden müssen. Sei dies geschehen, so könnten sich die Vertragspartner auf Wettbewerbsmärkten ausreichend selbst wehren, sei es, daß sie in Verhandlungen eine Änderung der Konditionen erreichten, sei es, daß sie ohne Verhandlungen vom Vertragsschluß einfach Abstand nehmen. In den Kategorien dieses Modells argumentiert der $\mathrm{BGH}$ in dem Annuitätendarlehens ${ }^{-36}$ und Wertstellungs-Urteil ${ }^{37}$, wenn er fordert, daß AGB-Klauseln so zu gestalten sind, daß Kunden die benachteiligende Wirkung einer Klausel ohne intensive Beschäftigung mit den $\mathrm{AGB}$ und ohne ergänzende Auskünfte deutlich werde.

Das andere Modell zeichnet sich dadurch aus, daß es nicht auf den Selbstschutz des informierten Kunden setzt, sondern auf beteronome, d. h. hoheitliche Kontrolle. Danach sollen Gerichte als neutrale Dritte für die Parteien verbindlich darüber entscheiden, ob die Konditionen unangemessen sind oder nicht. Es sind also nicht, wie normalerweise auf dem Wettbewerbsmarkt, die subjektiven Wertvorstellungen der Parteien sondern die Wertvorstellungen einer Kontrollinstanz maßgeblich.

Von daher stellt sich die Frage, ob dem AGBG und im besonderen dem $\$ 9$ AGBG das Informationsmodell oder das Modell heteronomer Kontrolle zugrunde liegt.

\section{1. $\int 9 A G B G$ als Instrument heteronomer Kontrolle des materiellen Vertragsinhalts}

a) Wortlaut

Der Wortlaut des $₫ 9$ AGBG deutet darauf hin, daß die Vorschrift dem Modell einer heteronomen Kontrolle verpflichtet ist. Dies ergibt sich aus \$9 II AGBG, wonach Kontrollmaßstab nicht die Wertvorstellung eines informierten Kunden oder sogar des konkreten Kunden, 
wenn er informiert gewesen wäre, ist, sondern das dispositive Recht, also eine von außen kommende Quelle materieller Vertragsgerechtigkeit. Allerdings deckt der Wortlaut des $\$ 9$ I AGBG durchaus die These, daß auch diejenigen Kunden unangemessen benachteiligt werden, die vor Vertragsschluß nicht angemessen über die Vertragskonditionen aufgeklärt worden sind und daher die für sie nachteiligen Vertragsangebote nicht ausschlagen konnten.

\section{b) Ratio des $₫ 9 A G B G$ \\ aa) Gesetzesmaterialien}

Der Regierungsentwurf zum AGBG ${ }^{38}$ sieht $\$ 9$ AGBG ausschließlich als Instrument heteronomer Kontrolle. Es ist nämlich an keiner Stelle von Information der Vertragspartner, sondern ausschließlich von der Verpflichtung des Verwenders die Rede, die Interessen der Beteiligten angemessen auszugleichen. Den gleichen Standpunkt nahm der Rechtsausschuß ein, demzufolge das Gebot eines notwendig objektiven angemessenen Interessenausgleiches die oberste Richtschnur für die Gestaltung vorformulierter Bedingungen ist ${ }^{39}$.

\section{bb) Bestätigung durch $\int 8 A G B G$}

Gerade weil die $\$ \mathbb{\int} 9 \mathrm{ff} A G B G$ Instrumente heteronomer Kontrolle darstellen, bedurfte es des $₫ 8$ AGBG.

Die ratio des $\$ 8$ AGBG kommt im Wortlaut der Vorschrift anerkanntermaßen nicht klar zum Ausdruck. Aufschlußreicher ist demgegenüber die Begründung zum Regierungsentwurf des $\mathrm{AGBG}^{+0}$. Dort heißt es, daß der Richter nicht befugt sein solle, über die Höhe des Entgelts und die Leistungsbeschreibung ein Angemessenheitsurteil abzugeben. Der Hintergrund hierfür ist, wie in der Literatur zu Recht verbreitet vertreten wird, daß in Hinblick auf Preis und Hauptleistung keine präzisen rechtlichen Angemessenheitsmaßstäbe existieren und existieren kön$n^{41}$. Mangels Einfühlungsmöglichkeit des Richters in die konkrete Marktsituation können objektive Maßstäbe, die marktwirtschaftliche Prinzipien nicht außer Kraft setzen, nicht so berechenbar sein, daß Preis und Hauptleistung ohne konkretisierende Parteivereinbarung einklagbar und vollstreckbar wären. Eine richterliche Kontrolle der Preise und

38 Begründung zum Regierungsentwurf, Bundestags-Drucksache 7/3919 S. 22.

39 Bundestags-Drucksache 7/5422 S. 9.

to Bundestags-Drucksache 7/3919 S.22.

+1 Vgl. Canaris, NJW 1987, 609, 613; H.P. Westermann in: 10 Jahre AGB-Gesetz (1988), S. 135, $136 \mathrm{ff}$ mit Nachweisen. 
ihres Verhältnisses zu den Hauptleistungen kann und soll daher nur in den Extremfällen der $\mathbb{\$} \$ 138 \mathrm{BGB}, 22 \mathrm{GW}$ B stattfinden.

Diese Beschränkung der richterlichen Kontrollrechte wäre nicht nachvollziehbar, wenn es im Rahmen des $\$ 9$ AGBG auch um eine ausreichende Information des Kunden bei Vertragsschluß ginge; denn diese Information ist gleichermaßen unproblematisch bei Preis, Hauptleistung und Nebenbestimmungen des Vertrages möglich. Daß ein Schutzbedürfnis der Vertragspartner in demselben Umfang wie z. B. in Hinblick auf vertragliche Haftungsregeln auch in Hinblick auf Preise und Hauptleistungsbeschreibungen bestehen kann, läßt sich nicht bestreiten. Im Gegenteil existiert in Hinblick auf Preis und Hauptleistung ein wesentlich intensiveres Schutzbedürfnis. Sind nämlich z. B. Bestimmungen, die unmittelbar den Preis oder die Hauptleistung betreffen, in AGB enthalten, so wird sie der Kunde bei Vertragsschluß typischerweise nicht zur Kenntnis nehmen. Die Kunden werden daher normalerweise ihre Entscheidung über die Angemessenheit von Leistung und Gegenleistung ohne Berücksichtigung dieser Nebenpunkte treffen. Nur zu leicht können sie deshalb insgesamt günstigere konkurrierende Angebote vernachlässigen. Vor allem können sie deshalb keinen Wettbewerbsdruck ausüben.

Dem kann man nicht entgegenhalten ${ }^{42}$, daß der Kunde auf die Information über den Inhalt von AGB, seien sie auf den Preis oder die Hauptleistung, seien sie auf sonstige Vertragskonditionen bezogen, nicht besonders intensiv angewiesen sei. Er habe nämlich ohnehin in der Regel keine Chance, ihm nicht genehme Punkte „wegzuverhandeln“. Auf das „Wegverhandeln-Können“ kommt es indessen nicht an, sondern allein darauf, daß auf dem Markt Wettbewerb herrscht und der Kunde den Vertragsschluß ablehnen kann, weil ihm persönlich bestimmte Konditionen zu nachteilig erscheinen. Der Wettbewerbsdruck entspringt entscheidend der Möglichkeit des Kunden, vom Vertrag Abstand zu nehmen und/oder auf Konkurrenten auszuweichen, nicht der Möglichkeit, bessere Vertragskonditionen herauszuhandeln. Dieser Wettbewerbsdruck wird dort eliminiert und der Kunde dort in erhöhtem Maß der Gefahr von Mißbräuchen ausgesetzt, wo der Kunde bei Einmalkontakten erst nach Vertragsschluß mit in AGB versteckten Preisbestimmungen konfrontiert wird oder wo er sie auch bei Mehrfachkontakten wegen der Art der Abrechnung normalerweise gar nicht bemerkt. Von daher ist es sehr verständlich, daß die Rechtsprechung, wie H.P. Westermann ${ }^{43}$ herausgearbeitet hat, den $₫ 8$ AGBG restriktiv interpretierte, um der

42 So aber Canaris, NJW 1987, 609, 613.

4. Fn. 41 S. 145 ff. 
Schutzbedürftigkeit der Kunden Rechnung zu tragen. Die Rechtsprechung hat das Ziel sicherlich richtig erkannt. Um das Ziel zu erreichen, muß $\$ 8$ AGBG jedoch nicht restriktiv ausgelegt werden. Man muß nur sehen, daß der Umstand, daß der Kunde in AGB mit einer Preis- oder Hauptleistungsregelung unvorbereitet oder gar irreführend konfrontiert wird, nicht eine Frage des $₫ 9$ AGBG sondern der $\$ \$ 2$ ff AGBG ist.

\section{cc) Benachteiligung durch unklare $A G B$ als Problem der Angemessenheit}

Damit soll nicht gesagt sein, daß im Rahmen des $\$ 9$ AGBG keine Informationsaspekte einfließen dürften. So ist es für die Frage, ob ein einseitiges Bestimmungsrecht des AGB-Verwenders angemessen oder unangemessen ist, sicherlich von erheblicher Bedeutung, daß der Kunde die rechtmäßige Ausübung des Bestimmungsrechts kontrollieren kann. $\mathrm{Da}$ es im Sinn des $\$ 9$ AGBG liegt, den Vertragspartner vor dem Effekt objektiv unangemessener Konditionen zu schützen, muß es auch gleichgültig sein, ob der Vertragspartner deshalb unangemessen benachteiligt wird, weil sich eine Klausel nach sachgerechter Auslegung nicht mehr im Rahmen eines angemessenen Interessenausgleichs hält oder weil der Vertragspartner das Klauselwerk nach Vertragsschluß nicht zu durchschauen vermag. Selbst wenn die einzelnen Klauseln durchwegs inhaltlich angemessen sind, so besteht doch bei $A G B$, die unzulänglich gegliedert, übermäßig umfangreich und auch sonst schwer verständlich sind, die große Gefahr, daß der Kunde mit unangemessenen Forderungen konfrontiert wird. Zwar erhält der Kunde durch $₫ 5$ AGBG einigen Schutz. Diesen Schutz hält die Rechtsprechung, zumal sie das Instrument des $\ 5$ AGBG äußerst zurückhaltend einsetzt ${ }^{44}$, bei verworrenen AGB zu Recht für nicht ausreichend. Der Kunde, gegen den der AGBVerwender gestützt auf seine AGB Ansprüche erhebt, muß ja überhaupt erst einmal von der Existenz des $\$ 5$ AGBG wissen. Selbst wenn er intuitiv dessen Existenz unterstellt, wird er nur zu leicht vor der Aufgabe resignieren, sich durch umfangreiche verworrene AGB hindurchzuwühlen. Er wird häufig lieber die Ansprüche seines Vertragspartners ohne nähere AGB-Kontrolle erfüllen. Wenn die Rechtsprechung also in diesen Fällen typische Desinformation des Vertragspartners zum Anlaß nimmt, diese Klauseln in Bausch und Bogen als unangemessen zu behandeln, so letztlich deswegen, weil derartige Klauseln leicht faktisch unangemessen eingesetzt werden können und der Verdacht groß ist, daß sie tatsächlich auch so eingesetzt werden ${ }^{45}$. Neben die rechtliche inhaltli-

t+ Siehe Fn. 10, 11.

t5 So im Ergebnis auch Staudinger/Schlosser, BGB (12. Aufl.), $₫ 3$ AGBG Rdn. 2. 
che Benachteiligung tritt somit die unmittelbare große Gefahr einer faktischen Benachteiligung wegen Unkenntnis der Rechtslage.

Insoweit orientiert sich die Rechtsprechung in den Extremfällen verworrener AGB an einem Informationsmodell. Dieses Informationsmodell ist jedoch nicht auf Informationen bei Vertragsschluß, sondern auf Informationen nach Vertragsschluß bezogen. Das ist für die Qualität der vom AGB-Verwender zu liefernden Informationen von großer Bedeutung. Nach Vertragsschluß ist nämlich davon auszugehen, daß der Kunde im Fall von Streitigkeiten die AGB eingehender studiert, um seine Rechte und Pflichten zu ermitteln. In dieser Phase kann und wird er in das Studium der AGB bedeutend mehr Zeit und Mühe investieren als vor Vertragsschluß, weil er nun dazu einen konkreten Anlaß hat. In

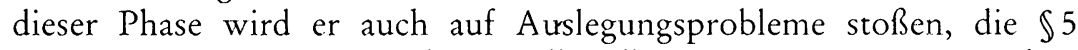
AGBG zu seinen Gunsten lösen soll. Selbst $₫ 5$ AGBG erspart es dem Kunden jedoch nicht, daß er die Klauseln vom Wortlaut ausgehend, nach der Rechtsprechung ${ }^{46}$ sogar zum Teil unter Berücksichtigung der systematischen Stellung der Klausel, deren Sinn und Zweck und deren Entstehungsgeschichte, wie ein verständiger Durchschnittskunde auslegt ${ }^{47}$. Weil vom Kunden derart weitgehende Auslegungsanstrengungen erwartet werden, brauchen selbst umfangreiche AGB nicht das Informationsniveau von Gesetzestexten zu übersteigen. Eine relevante Gefährdung des Kunden ist daher vom Standpunkt nach Vertragsschluß erst dann zu besorgen, wenn sich ein verständiger Durchschnittskunde mit den AGB trotz eingehender Beschäftigung nicht zurechtfindet. Erst dann sind sie als objektiv unangemessen zu behandeln.

In den Annuitätendarlehens ${ }^{-{ }^{8}}$ und Wertstellungsurteilen ${ }^{\text {t9 }}$ hat der BGH dagegen den wesentlich höheren Informationsbedarf des Kunden vor Vertragsschluß im Auge. Fordert man, daß der Kunde schon bei Vertragsschluß ausreichend informiert ist, so ist es angesichts der typischen Eile von Vertragsverhandlungen konsequent zu verlangen, daß Nachteile ohne intensive Beschäftigung mit den AGB erkennbar sind. Sind diese Anforderungen nicht erfüllt, so resultiert daraus jedoch nicht notwendig eine nach heteronomen Maßstäben objektive Benachteiligung, sofern die Klauseln inhaltlich angemessen sind und ihr Inhalt bei intensiverer Beschäftigung mit ihnen nach Vertragsschluß erkennbar ist ${ }^{50}$.

46 Siehe Fn. 10, 11.

47 Begründung zum CDU/CSU-Gesetzentwurf zum AGBG, Bundestags-Drucksache $7 / 3200$ S. 10.

ts Siehe Fn. 1.

+9 Siehe Fn. 2 .

so A. A. Niedenführ (Fn.6) S.110ff; Köndgen (Fn.27), die in Schwierigkeiten geraten, wenn außerhalb der AGB Informationen gegeben werden. Niedenführ (Fn.6) 
Dem Interesse der Vertragspartner, schon bei Vertragsschluß die sie benachteiligenden Klauseln zu kennen, um so besser individuelle Präferenzen verfolgen und Wettbewerbsdruck ausüben zu können, kann also nicht im Rahmen des $\$ 9$ AGBG Rechnung getragen werden. Diesem Informationsbedürfnis sind vielmehr die $\$ \$ 2,3$ AGBG gewidmet.

\section{Information als Schutzinstrument im Rabmen der $\iint 2$ ff $A G B G$}

Während die $\mathbb{S} 9 \mathrm{ff} A G B G$ im Wege hoheitlicher Korrektur objektiv inhaltlich unangemessene $A G B$ oder $A G B$, die inhaltlich unangemessen verwandt werden können, ausmerzen sollen, ist es Aufgabe der $\mathbb{\$} 2 \mathrm{ff}$ AGBG, das Informationsbedürfnis der Vertragspartner vor Vertragsschluß sicherzustellen. Weil es nur um Information geht, erstreckt sich der Schutz der $\$ \mathbb{S} 2 \mathrm{ff}$ A GBG auch auf Preis- und Hauptleistungsbestimmungen, da hier inhaltliche Angemessenheitsmaßstäbe keine Rolle spielen.

\section{a) Ratio und Tragreite des $\ 2 A G B G$}

Der Begründung zum Regierungsentwurf ${ }^{51}$ zufolge sollte $\$ 2$ AGBG dem Zweck dienen, den Vertragspartner über die Tragweite seiner Erklärungen bei Vertragsschluß zu informieren. Der Vertragspartner sollte die Tragweite seiner eigenen Erklärungen ermessen und sich gegebenenfalls gegen unerwünschte oder gar unbillige Bedingungen zu wehren in der Lage sein. Der Regierungsentwurf wollte sich damit bewußt von der Rechtsprechung absetzen, die eine Einbeziehung von AGB kraft „Wissen-Müssens“ des Vertragspartners bejahte ${ }^{52}$. Obwohl der historische Gesetzgeber den Informationsstand der potentiellen Vertragspartner vor Vertragsschluß verbessern wollte, konnte auch er sich nicht der Tatsache verschließen, daß AGB, auch wenn sie dem Vertragspartner bei Vertragsschluß in die Hand gedrückt werden, üblicherweise nicht gelesen werden. Es wird daher in der Begründung des Regierungsentwurfes ${ }^{53}$ betont, daß die in $\$ 2$ AGBG geregelte Einbeziehungsabrede nur den Charakter eines globalen Verweises auf und einer globalen Unterwerfung unter eine im einzelnen nicht ausge-

S. 115 will in diesen Fällen mit dem Argument arbeiten, daß wegen der Existenz des $₫ 3$ AGBG hier ein Bedarf zur Anwendung des $₫ 9$ AGBG fehle. Das Entscheidende ist, daß mangelnde Information bei Vertragsschluß nicht notwendig eine materielle Schlechterstellung des Kunden verursacht, sondern nur die Gefahr einer Schlechterstellung. Diese Gefahr ist angesichts der Tatsache, daß AGB ohnehin nicht gelesen werden, so gering, daß das Gesetz nicht jeden Informationsmangel als Benachteiligung qualifizieren mußte.

51 Bundestags-Drucksache 7/3919 S. 17.

52 Bundestags-Drucksache 7/3919 S. 17.

53 Bundestags-Drucksache 7/3919 S. 19. 
handelte, typisierte Regelung enthalte. Die in $\$ 2$ AGBG festgelegten, verhältnismäßig geringen Anforderungen seien für das Zustandekommen der Einbeziehungsabrede unter anderem deshalb vertretbar, weil der den AGB unterworfene Vertragsteil gemäß $\$ 3$ AGBG darauf vertrauen könne, daß sich die einzelnen Regelungen im großen und ganzen im Rahmen dessen bewegten, was nach den Umständen bei Abschluß des Vertrages erwartet werden könne ${ }^{54}$.

Im Einklang mit den Intentionen des historischen Gesetzgebers haben daher Literatur und Rechtsprechung das Informationsniveau im Rahmen des $\$ 2$ AGBG richtigerweise nie auf eine Höhe hinaufgeschraubt, auf dem potentiellen Vertragspartnern die benachteiligende Wirkung sämtlicher Klauseln ohne intensive Beschäftigung deutlich wird. Der Hinweisobliegenheit in $\$ 2$ I Nr. 1 AGBG wird vielmehr lediglich die Funktion einer Warnung zugeschrieben ${ }^{55}$. Eine Unterrichtung über den Inhalt der AGB ist mit dem Hinweis auf die AGB nicht verbunden. Gleiches gilt für den Aushang der AGB. An die Möglichkeit zur zumutbaren Kenntnisnahme ( $\$ 2 \mathrm{I} \mathrm{Nr} .2 \mathrm{AGBG})$ werden keine hohen Anforderungen gestellt. Ulmer ${ }^{56}$ betont, daß sich der Gesetzgeber entgegen ursprünglichen Intentionen, immer die Übermittlung der AGB zu fordern, mit der Möglichkeit der zumutbaren Kenntnisnahme begnügt habe ${ }^{57}$. Demgemäß soll auch nicht darauf hingewiesen werden müssen, daß und wo die Möglichkeit zur Einsichtnahme in die AGB besteht ${ }^{58}$. Bei telefonischen Vertragsschlüssen genügt sogar der Hinweis auf die AGB. Es sei hier Sache des Kunden, ob er sich die AGB durchsagen lasse oder darauf verzichtet. Schlosser ${ }^{59}$ geht einen Schritt weiter. Er weist einleuchtend darauf hin, daß AGB-Gesetz habe nicht utopischerweise erwartet, Letztverbraucher würden sich in ihrer Entscheidung über den Vertragsschluß durch den Inhalt der AGB motivieren lassen ${ }^{60}$. Hätte das Gesetz. dies angenommen, so hätte es vorsehen müssen, daß die $A G B$ dem Vertragspartner so rechtzeitig vor Vertragsschluß zur Kenntnis gebracht werden, daß er sie noch durcharbeiten kann. Einzig realistische Funktion des $₫ 2$ AGBG könne es daher nur sein, den Vertragspartner über die Geltung von $A G B$ zu informieren ${ }^{61}$ und ihn im Konfliktfall und zu seiner Orientierung während der Vertragsabwicklung in die Lage zu

\footnotetext{
5t Bundestags-Drucksache 7/3919 S. 19.

55 Soergel/Stein, BGB (11. Aufl.), $\$ 2$ AGBG Rdn. 2.

56 Ulmer in: Ulmer/Brandner/Hensen, AGBG (5. Aufl.), $\$ 2$ AGBG Rdn. 45.

57 Ebenso Soergel/Stein (Fn. 55) $\$ 2$ AGBG Rdn. 2.

5s Ulmer (Fn. 56) \$2 AGBG Rdn. 47.

59 Staudinger/Schlosser (Fn. 45) \2 AGBG Rdn. 26.

60 Insoweit zustimmend Soergel/Stein (Fn. 55) \$2 AGBG Rdn. 2.

${ }_{61}$ Niedenführ (Fn. 6) S. 97.
} 
versetzen, mühelos einen Text der $\mathrm{AGB}$ zur Verfügung zu haben. Schlosser ${ }^{62}$ will deshalb auch die Kenntnisverschaffung nach Vertragsschluß genügen lassen. Selbst wenn man diesen Schritt, der sich weder mit dem Wortlaut des $\$ 2$ AGBG noch mit den Vorstellungen des historischen Gesetzgebers vereinbaren läßt, nicht mitmacht, so läßt sich doch insgesamt nicht bestreiten, daß $\$ 2 \mathrm{AGBG}$ nicht nachdrücklich und realistisch eine Information des potentiellen Vertragspartners erzwungen hat, die diesen ausreichend über die Nachteile des Geschäfts infolge der AGB-Unterwerfung unterrichtet.

Dieser These steht die Forderung nach Verständlichkeit und Lesbarkeit der $\mathrm{AGB}^{63}$ nicht entgegen; denn im Einklang mit der Bemerkung im Regierungsentwurf ${ }^{6+}, \mathrm{da} ß$ an die Einbeziehungsvoraussetzungen verhältnismäßig geringe Anforderungen gestellt werden, müssen die AGB grundsätzlich nur für jemanden verständlich sein, der sie nach Vertragsschluß durcharbeitet. Die Verständlichkeit wurde daher vor den Annuitätendarlehens ${ }^{-65}$ und Wertstellungsentscheidungen ${ }^{66}$ nur in den Fällen verneint, in denen die AGB unübersichtlich aufgebaut waren, keine Gliederung erkennen ließen oder einen gegenüber der Bedeutung des Geschäfts und dem sachlichen Regelungsgehalt unverhältnismäßigen Umfang aufwiesen ${ }^{67}$. Es wurde kaum jemals gefordert, daß die AGB in der realen Vertragsschlußsituation ohne intensive Beschäftigung verständlich sein müßten ${ }^{68}$.

Als Zwischenergebnis kann daher festgehalten werden, daß AGB gemäß $\ 2$ AGBG auch dort Vertragsbestandteil werden, wo die Kunden in der typischen Situation vor Vertragsschluß nicht ohne weiteres in der Lage sind, die den AGB entspringenden Nachteile zu durchschauen.

\section{b) Die Informationsfunktion des $\mathbb{3} A G B G$}

$\$ 3$ AGBG statuiert unmittelbar keine Informationsobliegenheit, wohl aber mittelbar. Weil Bestimmungen in AGB, mit denen der Vertragspartner nicht zu rechnen braucht, nicht Vertragsbestandteil werden, muß der AGB-Verwender, der sie zum Vertragsbestandteil erheben will,

${ }_{62}$ Siehe Fn. 59; a. A. freilich die ganz h. M., die in dieser Auslegung ein Zeichen des Fatalismus sieht (Soergel/Stein [Fn. 55] $\$ 2$ AGBG Rdn. 17).

${ }^{63}$ Begründung zum Regierungsentwurf des AGBG, Bundestags-Drucksache 7/3919 S. 18.

it Bundestags-Drucksache 7/3919 S. 19.

${ }^{65}$ Siehe Fn. 1.

66 Siehe Fn. 2.

67 Ulmer (Fn. 56) $\$ 2$ Rdn. 52 m. umfassenden Nachweisen; weitergehend Soergel/ Stein (Fn. 55) $\$ 2$ AGBG Rdn. 19.

${ }_{68}$ Anders Wolf (Fn. 7) $\$ 2$ Rdn. 29. 
die Vertragspartner entsprechend den typischen Gegebenheiten bei Vertragsschluß so informieren, daß sie mit dem Inhalt der AGB rechnen müssen ${ }^{69}$. Das AGBG orientiert diese mittelbare Informationsobliegenheit realistisch an dem Erfahrungssatz, daß AGB in der Regel vernünftigerweise vor Vertragsschluß nicht gelesen werden ${ }^{70}$ und die Statuierung umfassender Informationspflichten das Informationsdefizit der Kunden nicht beseitigt ${ }^{71}$. In dieser Situation fand sich der Gesetzgeber nicht bereit, es bei den bloßen typischerweise nicht genutzten Informationsmöglichkeiten der potentiellen Vertragspartner gemäß $\$ 2$ AGBG zu belassen und im übrigen auf die Angemessenheitskontrolle der $\int \$ 9 \mathrm{ff}$ AGBG zu setzen. Vielmehr forderte er als Voraussetzung der Verbindlichkeit der $A G B$, daß der Vertragspartner über Inhalt und Tragweite der $A G B$ informiert ist oder informiert sein muß, allerdings nur im großen und ganzen, d. h. nicht in allen Details ${ }^{72}$.

Aus der Formulierung des $\$ 3$ AGBG, die die Ungültigkeit der Klausel an die „Ungewöhnlichkeit“ der Klausel knüpft, darf nun nicht hergeleitet werden, daß die Vertragspartner nicht überrascht werden können und die Klausel ihnen daher nicht transparent gemacht zu werden braucht, wenn die Klausel in einer Branche für den Vertragstyp, für den die Klausel verwandt wird, weit verbreitet, ja üblich ist ${ }^{73}$. Die weite Verbreitung einer Klausel, sogar die Üblichkeit einer Klausel ist nicht absolut entscheidend. In Teilen der Kommentarliteratur wird zu Recht anerkannt, daß ohne besondere Informationen eine Klausel selbst dort überraschend sein kann, wo sie branchenüblich ist ${ }^{74}$. Diese Lehre befindet sich im Einklang mit dem Willen des historischen Gesetzgebers. Aus der Begründung zum Regierungsentwurf ${ }^{75}$ ergibt sich nämlich, daß es nicht primär auf die Üblichkeit oder Ungewöhnlichkeit einer Klausel ankommt. Vielmehr sei in erster Linie auf die verständige Erwartungshaltung des Vertragspartners abzuheben ${ }^{76}$. So heißt es: „Das Überraschungsmoment muß sich aus einer deutlichen Diskrepanz zwischen der durch die Umstände bei Vertragsschluß begründeten Erwartung des

(1) Ulmer (Fn. 10) $\$ 3$ Rdn. 1; Staudinger/Schlosser (Fn. 45) $\$ 3$ AGBG Rdn. 2.

70 Begründung des Regierungsentwurfs zum AGBG, Bundestags-Drucksache 7/3919 S. 19; Wolf (Fn. 7) \$3 Rdn. 1; Münchener Kommentar-Kötz (Fn. 12) $\$ 3$ Rdn. 1.

7 Dauner-Lieb (Fn. 7) S. 73; ebenso jetzt zutreffend Köndgen (Fn. 27).

72 Bundestags-Drucksache 7/3919 S. 19.

73 So aber Canaris, NJW 1987, 609, 610; Kollhosser, ZIP 1986, 1429, 1433 f; BGH, NJW 1985, 848, 849; 1981, 117, 118.

${ }^{7}$ Wolf (Fn. 7) $\$ 3$ Rdn. 14; Ulmer (Fn. 10) $\$ 3$ Rdn. 20; anders Rdn. 43; ebenso Köndgen, NJW 1987, 160, 164.

75 Bundestags-Drucksache 7/3919 S. 19.

7o Ebenso klar Wolf (Fn. 7) $₫ 3$ Rdn. 3, 11, $14 \mathrm{f}$; Münchener Kommentar-Kötz (2. Aufl.), $\$ 3$ AGBG Rdn. 3 ff. 
anderen Vertragsteils und dem tatsächlichen Inhalt der AGB ergeben. Eine spezifizierte Aufzählung der Einzelumstände, die als Vertrauensgrundlage maßgeblich sein können, erscheint, wenngleich vielleicht wünschenswert, nicht möglich“77. Der Begriff „so ungewöhnlich“, der in $\$ 3$ AGBG verwandt wird, soll demnach lediglich die Stärke der Diskrepanz zwischen Erwartungshaltung und Realität verdeutlichen, nicht aber soll dieser Begriff besagen, daß eine Diskrepanz zwischen der Erwartungshaltung verständiger Vertragspartner und dem auf dem Markt Gewöhnlichen immer unerheblich sei. Auch verständige Vertragspartner müssen nicht immer wissen, was auf einem Markt auf dem AGB-Sektor üblich ist.

Aus $₫ 3$ AGBG ergibt sich mithin, daß nicht immer die Obliegenheit besteht, den Kunden die benachteiligende Wirkung einer Klausel so zu verdeutlichen, daß sie bei Vertragsschluß ohne intensive Beschäftigung mit den AGB durchschaut werden kann. Vielmehr existiert diese Obliegenheit nur in Hinblick auf solche Klauseln, mit denen der Vertragspartner bei weitem nicht zu rechnen brauchte.

Zur Kategorie dieser überraschenden Klauseln zählen typischerweise nicht Bestimmungen, die die Modalitäten der Vertragsabwicklung den Besonderheiten des Geschäfts anpassen oder Risiken verteilen, weil Vertragspartner, die auf die Einbeziehung von AGB aufmerksam gemacht worden sind, mit derartigen Klauseln rechnen und ihre Vertragsentscheidungen auf dieser Grundlage treffen.

Anders ist die Situation jedoch dort, wo Klauseln unmittelbar den Gegenstand der Leistung oder die Vergütung berühren. Hier gehen die potentiellen Kunden normalerweise davon aus, daß alle für die Bestimmung der Leistung und des Preises wesentlichen Faktoren auf den Tisch gelegt werden. Preis und Leistung stehen nämlich im Mittelpunkt ihrer Entscheidungen. Ihr Interesse konzentriert sich daher typischerweise auf diese Daten. Weil die Anbieter um dieses Interesse der Nachfrager wissen, werden die Nachfrager auch typischerweise über die Leistung und die Preise eingehend informiert; denn Preis und Leistung sind die stärksten Wettbewerbsparameter. Die Nachfrager erwarten deshalb erfahrungsgemäß nicht, in den AGB ohne besondere Verdeutlichung Bestimmungen zu finden, die den Leistungsinhalt oder die Preise modifizieren. So betreffen die in der Begründung zum Regierungsentwurf ${ }^{78}$ genannten Beispiele für überraschende Klauseln bezeichnenderweise durchwegs Fälle, in denen Vergütungspflichten im Rahmen von AGB erhöht wurden.

77 Bundestags-Drucksache 7/3919 S. $19 \mathrm{f}$.

78 Bundestags-Drucksache 7/3919 S. 19. 
Ähnlich war die Sachlage im Annuitätendarlehens-Urteil ${ }^{79}$. Die Zinsberechnungsmethode erhöhte die Gesamtleistung, die der Darlehensnehmer für die Nutzung des Kredits schuldete. Diese Erhöhung trat immer und bei jedem Kunden ein; sie hing also nicht von in der Zukunft liegenden Risikofaktoren oder ähnlichen Umständen ab. Da Darlehensnehmer typischerweise davon ausgehen, daß der ihnen genannte Zinssatz vom effektiven Zinssatz nur in dem Umfang abweicht, in dem weitere ihnen offenbarte Vergütungselemente, wie z.B. ein Disagio oder Bearbeitungsgebühren, eine Rolle spielen, war eine nicht hinreichend transparente Zinsberechnungsmethode, die zu effektiven Zinserhöhungen führte, überraschend. Dies gilt jedenfalls für solche Zinsberechnungsmethoden, bei denen sich der Umfang der Erhöhung des Vertragszinses bei Vertragsschluß ohne jede Wahrscheinlichkeitsüberlegungen quantifizieren läßt.

Auf die Üblichkeit des Erhöhungseffekts ${ }^{80}$ kommt es demgegenüber nur an, wenn er wegen der Üblichkeit in etwa in das Bewußtsein der Vertragspartner gedrungen ist. Man könnte zwar argumentieren, daß sich in Fällen, in denen eine Klausel mit preiserhöhendem Effekt allgemein verbreitet ist, der Wettbewerb auf der Basis dieser Klausel abspiele. Die Marktergebnisse würden daher nicht verfälscht, selbst wenn die Vertragspartner bei Vertragsschluß nicht ausreichend über den Preiserhöhungseffekt der Klausel informiert sind. In der Tat besteht in diesen Fällen auf den ersten Blick kein Informationsbedarf der Kunden. Bei näherem Zusehen läßt sich ein Informationsbedarf jedoch nur in solchen Fallgruppen verneinen, in denen nachweislich alle Anbieter die verdeckt benachteiligende Klausel verwandt haben und die Nachfrager ihre Nachfrageentscheidungen auch bei Kenntnis des Preiserhöhungseffekts nicht geändert hätten. Es darf also nicht zu erwarten gewesen sein, daß die Nachfrager auf andere Güter ausweichen. Dies wird man indessen kaum jemals allgemein bejahen können, da es im Kreis der Nachfrager immer Personen geben wird, deren Nachfrageentscheidung auf der Kippe steht und die bei geringsten Preiserhöhungen auf einen Vertragsschluß verzichten. Angesichts der zur Verfügung stehenden Informationsmöglichkeiten verbietet es der Schutz der Vertragspartner, die Interessen dieser Nachfragergruppe zu vernachlässigen.

Insgesamt kann daher festgestellt werden, daß der AGB-Verwender dem Vertragspartner benachteiligende Klauseln immer dann vor Vertragsschluß derart zu verdeutlichen hat, daß dieser sie ohne intensive Beschäftigung in ihrer Tragweite durchschauen kann, wenn sie ohne die

79 Siehe Fn. 1.

so Siehe oben bei Fn. 76 . 
Verdeutlichung überraschend wären. Für die Frage, ob Klauseln überraschend sind, kommt es nicht auf die Üblichkeit der Klauseln, sondern auf die Erwartungshaltung verständiger Kunden an. Je preis- und/oder hauptleistungsnäher Klauseln sind, um so eher sind sie ohne besondere Verdeutlichung überraschend ${ }^{81}$.

\section{Verbandsklage}

Die Ableitung des auf den Zeitpunkt des Vertragsschlusses bezogenen Transparenzgebots als Ausfluß des $\$ 3$ AGBG scheint allerdings zur Folge zu haben, daß Verbände ihre Klage nicht auf eine Verletzung des Transparenzgebots vor Vertragsschluß stützen können. Der Unterlassungs- und Widerrufsanspruch ist nämlich gemäß $\$ 13$ AGBG nur auf Verstöße gegen die $\$ \$ \$$ 9-11 AGBG bezogen. Die daraus resultierende Beschränkung der Verbandsklage erscheint jedoch in den Fällen sinnwidrig, in denen in typischer Weise Klauseln überraschend verwendet werden. Dies dürfte den BGH bewogen haben, das Transparenzgebot auch, soweit es auf die Phase vor Vertragsschluß bezogen ist, auf $\$ 9$ AGBG zu stützen. Dieser Umweg ist indessen nicht notwendig, wenn man die $\mathbb{S} 13 \mathrm{ff} A G B G$ auf typischerweise überraschende Klauseln analog anwendet ${ }^{82}$. Der Umstand, daß die Klauseln gemäß $\$ \$ 9 \mathrm{ff} A G B G$ unwirksam sind und gemäß $₫ 3$ AGBG nicht Vertragsinhalt werden, rechtfertigt keine Differenzierung; denn auch die Verhinderung der Einbeziehung soll den Vertragspartner vor subjektiv unausgewogenen Verträgen schützen. Gegen eine Analogie spricht allein der Umstand, daß es gemäß $₫ 3$ AGBG auf die konkrete Situation bei Vertragsschluß ankommt und daß diese Situation bei Vertragsschluß z. B. nicht im Unterlassungsurteil gemäß $₫ 17$ AGBG angegeben werden kann ${ }^{83}$. Dies braucht indessen nicht zu geschehen, wenn man im Rahmen der $\$ \$ 13 \mathrm{ff}$ AGBG nur solche Klauseln für gemäß $\$ 3$ AGBG unterbindbar erklärt, die ohne irgendeine Verdeutlichung verwandt werden. Es ist auch daran zu denken, daß die Vertragsschlußsituation im Untersagungsurteil neben der beanstandeten Klausel angegeben wird. Dies müßte letztlich auch dort erfolgen, wo eine Klausel gemäß $\$ 9$ AGBG wegen Intransparenz bei Vertragsschluß angegriffen wird. Dies zeigt deutlich das Annuitätendarlehens-Urteil ${ }^{\$ 4}$. Der BGH stellte nämlich auch im Rahmen des $\$ 9$

81 Ebenso i. E. Niedenfübr (Fn. 6) S. $151 \mathrm{f}$.

82 Lindacher in Wolf/Horn/Lindacher, AGBG (1.Aufl.), $\$ 13$ Rdn. 29; Palandt/ Heinrichs (Fn. 12) $\$ 13$ AGBG Anm. 2 b; a. A. BGH, NJW-RR 1987, 45, 46; Hensen in Ulmer/Brandner/Hensen, AGBG (5. Aufl.), $\$ 13$ Rdn. 8.

8.3 BGH, NJW-RR 1987, 45, 46.

st Siehe Fn. 1. 
AGBG auf die konkrete Vertragsschlußsituation ab; denn er führte aus, daß die Klausel aus sich heraus nicht immer unangemessen sei. Vielmehr wäre die vom Wortlaut her identische Klausel angemessen gewesen, wenn die Kreditinstitute den Effektivzins im Sinn der PreisangabenVO offenbart hätten.

\section{Ergebnisse}

1. Es ist zwischen den auf die Vertragsschlußsituation und den auf die Situation nach Vertragsschluß bezogenen Transparenzanforderungen zu unterscheiden.

2. AGB müssen so transparent sein, daß sie nach Vertragsschluß bei eingehenderem Studium in ihrer Tragweite durchschaut werden können. 3. Dagegen brauchen AGB nicht immer derart transparent zu sein, daß Vertragspartner schon vor Vertragsschluß ohne intensive Beschäftigung mit den Klauseln sämtliche auf sie zukommenden Nachteile erkennen können.

4. Eine derartige Transparenz ist nur dort erforderlich, wo die Vertragspartner nicht in etwa mit den sie benachteiligenden Klauseln rechnen müssen. Je preis- und leistungsnäher die Klauseln sind, um so transparenter müssen sie bereits vor Vertragsschluß sein. Dort, wo wegen $\mathbb{8} 8$ AGBG keine Angemessenheitskontrolle eingreift, muß volle Transparenz vor Vertragsschluß herrschen. Ein Verstoß gegen das Transparenzgebot vor Vertragsschluß führt zur Anwendbarkeit des $₫ 3$ AGBG. Typische Verstöße gegen $\ 3$ AGBG können im Weg der Verbandsklage verfolgt werden. 